\title{
Pancreatic Neuroendocrine Tumor G1
}

National Cancer Institute

\section{Source}

National Cancer Institute. Pancreatic Neuroendocrine Tumor G1. NCI Thesaurus. Code C95584.

A low grade well differentiated tumor with neuroendocrine differentiation that arises from the pancreas. The mitotic count is less than 2 per $10 \mathrm{HPF}$ and/or the Ki67 index is equal or less than $2 \%$. 